\title{
1 Theoretischer Teil
}

\subsection{Daten zur Nutzung interaktiver und nicht-interaktiver Bildschirmmedien}

Medienwirkungsforschung ist unmittelbar verknüpft mit der Frage nach der "Wahrscheinlichkeit, auf ein entsprechendes Wirkpotential zu treffen" (Groebel \& Gleich, 1993, S. 136). Eine Darstellung von Daten zur Nutzungshäufigkeit von Bildschirmmedien sowie zur Bedeutung gewalthaltiger Angebote soll deshalb den Ausgangspunkt dieser Arbeit bilden. Hiermit wird veranschaulicht, an welchen gesellschaftlichen Problemkontext diese Arbeit anknüpft. Der Überblick konzentriert sich dabei auf die beiden in dieser Untersuchung behandelten und hinsichtlich der Gewaltmediendebatte prominentesten Bildschirmmedien: Das Computerspiel als interaktives und das Fernsehen als nicht-interaktives Medium.

\subsubsection{Geräteausstattung und Nutzungszeiten}

Mit dem Einzug des Fernsehers in die privaten Haushalte in der zweiten Hälfte des 20. Jahrhunderts und mit der in jüngerer Zeit zunehmenden privaten Verbreitung von Computer und Internet sind Bildschirmmedien zu einem wichtigen Bestandteil des alltäglichen Freizeiterlebens geworden (vgl. Huston \& Wright, 1998). ${ }^{2}$ Während die Nutzung linear-rezeptiver Bildschirmunterhaltung im Sinne des klassischen "Fernsehens" jedoch inzwischen nahezu stagniert und in jüngeren Alterspopulationen sogar rückläufig ist (Feierabend \& Klingler, 2008), gewinnen interaktive Unterhaltungsmedien fortwährend an Bedeutung. ${ }^{3}$ So verfügten im Jahr 1999

2 In diesem Kapitel werden zur Darstellung der kindlichen und jugendlichen Medienumwelt aufgrund ihrer deutschlandweiten Repräsentativität maßgeblich die Kinder- und Jugendstudien KIM und JIM des Medienpädagogischen Forschungsverbundes Südwest herangezogen. In beiden Studienarmen werden seit 1998 (JIM) bzw. 1999 (KIM) Mediennutzungsparameter von Kindern im Alter zwischen 6 und 13 Jahren (KIM) und Jugendlichen im Alter von 12 19 Jahren (JIM) erhoben. Eine Sonderschwerpunktauswertung bietet zudem, wenngleich nicht mehr ganz aktuell, Daten zu Vorschulkindern aus dem Erhebungsjahr 2003 (Feierabend \& Mohr, 2004). Hier wurde eine deutschlandweit repräsentative Stichprobe von 245 Eltern zu ihren Kindern im Alter von zwei bis fünf Jahren befragt.

3 Nach dem AGF/GFK-Fernsehpanel, in welchem die Zeiten technisch-verhaltensbasiert und nicht mittels Fragebogen erfasst werden (vgl. GfK-Fernsehforschung, 2005), verbrachten dreibis fünfjährige Kinder 1992 täglich 66 Minuten mit dem Fernsehen, ein Wert der seither nur geringfügig und mit leichter Schwankungsbreite auf 73 Minuten im Jahr 2007 angestiegen ist. Für die Altersgruppen der Sechs- bis Neunjährigen sowie der Zehn- bis Dreizehnjährigen ist 
nur etwa 50 Prozent der Haushalte mit Kindern über eine Computerausstattung ( 47 \% Computer, 7 \% Notebook) und etwa 44 Prozent über eine Spielkonsole (vgl. Feierabend \& Klingler, 2000). Im Jahr 2008 sind hingegen 88 Prozent der Haushalte mit Kindern und 99 Prozent der Haushalte mit Jugendlichen mit mindestens einen stationären oder tragbaren Computer ausgestattet, etwa zwei Drittel verfügen über eine Spielkonsole (Feierabend \& Rathgeb, 2008, 2009 b).

Vor dem Hintergrund dieser Entwicklung sind Lebenswelten von Heranwachsenden heute mehr denn je auch Medienwelten (vgl. Hoppe-Graff \& Kim, 2002). Seit 1998 hat sich die Ausstattung mit Computern und Spielkonsolen im Jugendzimmer mehr als verdoppelt, etwa die Hälfte der Jugendlichen verfügt inzwischen über einen eigenen Internetanschluss. Die Ausstattungsquote mit Fernsehgeräten ist hingegen in den letzten zehn Jahren verhältnismäßig konstant geblieben (vgl. Abbildung 1).

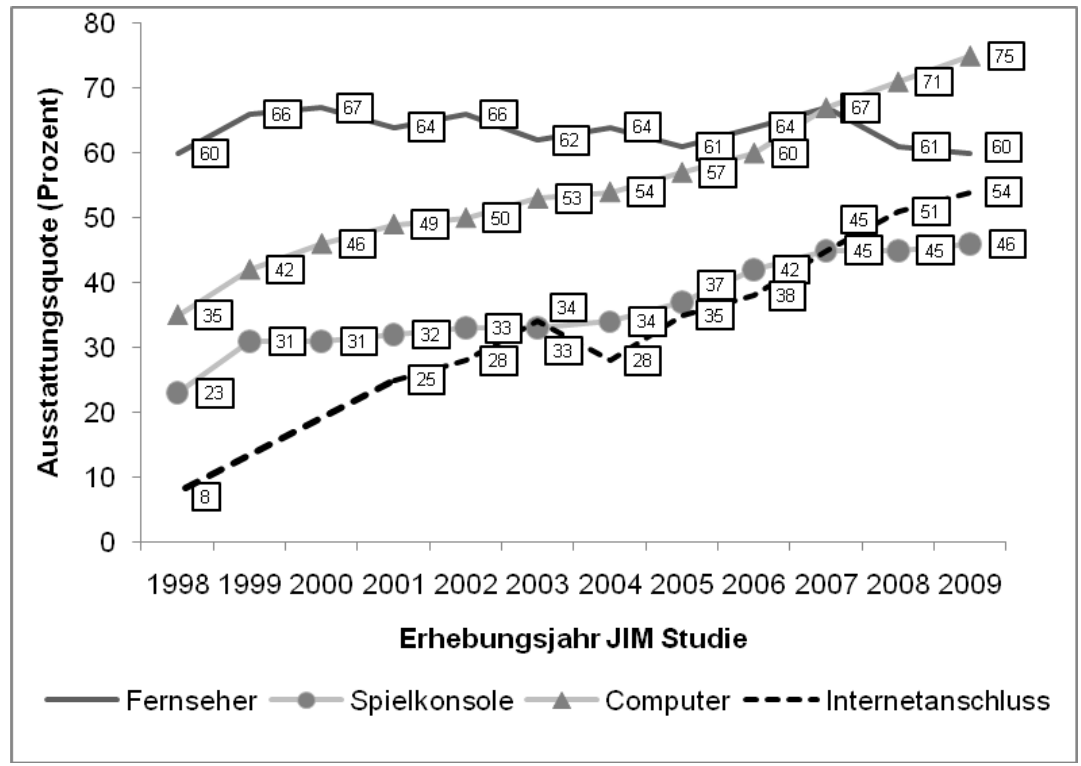

Abbildung 1. Ausstattung von Jugendzimmern (12-19-Jährige) mit Bildschirmmediengeräten und Internetzugang von 1998 bis 2008 (Feierabend \& Klingler, 1998, 1999, 2000, 2001, 2002, 2003; Feierabend \& Rathgeb, 2004, 2005, 2006, 2007, 2008, 2009 a) ${ }^{4}$

ein leichter Rückgang der Nutzungsdauer festzustellen. Nur für die Gruppe der über 14-Jährigen ist ein Anstieg der Fernsehnutzungsdauer von 168 Minuten im Jahr 1992 auf 223 Minuten im Jahr 2007 zu verzeichnen (vgl. Feierabend \& Klingler, 2008).

4 Für die Jahre 1998, 1999 und 2000 sind aus den einzelnen JIM-Studien keine Angaben zum Internetanschluss im Jugendzimmer zu entnehmen. Zum Zwecke der Veranschaulichung wur- 
Die Ausstattung mit eigenen Mediengeräten setzt bereits im Vorschulalter ein. Während jedoch von Vorschulkindern im Jahr 2003, dem aktuellsten Zeitpunkt zu welchem zu dieser Altersgruppe noch deutschlandweit repräsentative Daten vorliegen, weniger als 10 Prozent mit einem eigenen Fernseher, Spielkonsole oder Computer im Kinderzimmer ausgestattet waren (Feierabend \& Mohr, 2004), verfügen im Jahr 2008 Kinder im Alter von 6 - 13 Jahren zu 42 Prozent (Feierabend \& Rathgeb, 2009 b) und Jugendliche im Alter von 12 bis 19 Jahren zu 61 Prozent (Feierabend \& Rathgeb, 2008) über ein eigenes Fernsehgerät. Die Ausstattung mit Spielkonsolen steigt ebenfalls von 25 Prozent im Kindesalter auf etwa 45 Prozent im Jugendalter an, die Ausstattung mit Computern von 15 Prozent auf 71 Prozent (Feierabend \& Rathgeb, 2008). Eine aktuelle deutschlandweit repräsentative Schülerbefragung des Kriminologischen Forschungsinstituts Niedersachsen ermittelt für Jugendliche im Alter von 15 Jahren eine Fernseh- und Computerausstattung von jeweils 70 Prozent und eine Spielkonsolenausstattung von 46 Prozent (vgl. Rehbein, Kleimann, \& Mößle, 2009 a). Damit liegt die Ausstattung mit Computern inzwischen im Jugendalter etwa gleichauf mit der Fernsehausstattung, nach JIM-2008 wäre sogar von einer höheren Ausstattung mit Computern auszugehen (vgl. Tabelle 1).

Tabelle 1. National repräsentative Daten zur Bildschirmgeräteausstattung in verschiedenen Alterspopulationen

\begin{tabular}{lllllll}
\hline Autoren (Jahr) & Stichprobe / Erhebungsjahr & N & Alter & TV & Konsole & PC \\
\hline $\begin{array}{l}\text { Feierabend \& Mohr } \\
\begin{array}{l}\text { (2004) } \\
\text { KIM Sonderschwerpunkt }\end{array}\end{array}$ & $\begin{array}{l}245 \\
\text { (Eltern) }\end{array}$ & $2-3$ & $4 \%$ & $6 \%$ & $0 \%$ \\
\hline $\begin{array}{l}\text { Feierabend \& Rath- } \\
\text { geb (2009b) }\end{array}$ & KIM 2008 & $\begin{array}{l}1.206 \\
\text { (Mütter) }\end{array}$ & $6-13$ & $42 \%$ & $\begin{array}{l}25 \%(\mathrm{SK}) \\
46 \%(\mathrm{TK})\end{array}$ & $15 \%$ \\
\hline $\begin{array}{l}\text { Feierabend \& Rath- } \\
\text { geb (2008) }\end{array}$ & JIM & 1.208 & $12-19$ & $61 \%$ & $\begin{array}{l}45 \%(\mathrm{SK}) \\
41 \%(\mathrm{TK})\end{array}$ & $71 \%$ \\
\hline $\begin{array}{l}\text { Rehbein, Kleimann } \\
\text { \& Mößle (2009) }\end{array}$ & $\begin{array}{l}\text { Schülerbefragung } \\
\text { 2007 \& 2008 }\end{array}$ & 44.610 & 15 & $70 \%$ & $\begin{array}{l}46 \%(\mathrm{SK}) \\
50 \%(\mathrm{TK})\end{array}$ & $70 \%$ \\
\hline
\end{tabular}

Anmerkung. SK = stationäre Spielkonsole, $\mathrm{TK}=$ Tragbare Spielkonsole.

In Entsprechung zu den Medienausstattungsparametern ist gerade die Nutzung interaktiver Unterhaltungsangebote deutlich angestiegen. Obgleich der Computer - im Gegensatz zur Spielkonsole - neben dem Spielen eine inzwischen nahezu unüberschaubare Bandbreite möglicher Nutzungsformen aus den Bereichen Kom-

de für das Jahr 1998 die Haushaltsausstattung mit Internetanschlüssen in die Grafik einbezogen (vgl. Datenreport 2004: Zahlen und Fakten über die Bundesrepublik Deutschland, 2005, S. 139). 
munikation (z. B. Email, Chat, Foren), Information (z. B. Recherchearbeiten, Nutzung von Wissensseiten) und kreative Bearbeitung (z. B. Programmieren, Videobearbeitung) ermöglicht, ermittelt die KIM-Studie als die häufigste Computeraktivität, die mindestens einmal pro Woche von Jungen oder Mädchen im Alter von 6 - 13 Jahren ausgeübt wird, das Computerspielen (Feierabend \& Rathgeb, 2009 b). Während das Arbeiten für die Schule und die Nutzung von Lernprogrammen bei Mädchen eine vergleichbare Relevanz wie das Computerspielen einnehmen, besteht gerade bei Jungen eine deutliche Präferenz zugunsten des Computerspielens und des Surfens im Internet (Feierabend \& Rathgeb, 2009 b). Dies gilt in ähnlicher Weise für Jugendliche im Alter zwischen 12 und 19 Jahren: Auch hier ist das Computerspielen für männliche Jugendliche die wichtigste Computeraktivität. Jugendliche Mädchen verbringen hingegen die meiste Zeit am Computer mit dem Arbeiten für die Schule (Feierabend \& Rathgeb, 2008).

Die zunehmende Verbreitung der interaktiven Medien Spielkonsole und Computer und die große Bedeutung, die hierbei den Computerspielen zukommt, lässt einen deutlichen Anstieg der Computerspielnutzungszeit erwarten. Dies deutet auch die Entwicklung der Marktzahlen an: So wurden im Jahr 2000 noch etwa 33 Millionen Video- und Computerspiele in Deutschland verkauft, im Jahr 2008 waren es schon rund 56 Millionen. Entsprechend stieg der Umsatz der Spiele-Branche in Deutschland von 874 Millionen Euro im Jahr 2000 auf 1,56 Milliarden Euro im Jahr 2008 an (Bundesverband Interaktive Unterhaltungssoftware e.V., 2006, 2009). Da die KIM- und JIM-Studien keine durchschnittlichen Computerspielnutzungszeiten erfassen, werden im Folgenden vergleichende Daten der Befragungen neunter Schulklassen des Kriminologischen Forschungsinstituts Niedersachsen aus den Jahren 2000, 2005 und 2007/2008 herangezogen. Wenngleich die Erhebungsmethodik dieser Befragungen in einigen Details voneinander abweicht und Unterschiede zwischen den Messzeitpunkten vor diesem Hintergrund interpretiert werden müssen, vermitteln die Daten für beide Geschlechter einen deutlichen Anstieg der Nutzungsdauer (vgl. Abbildung 2). 


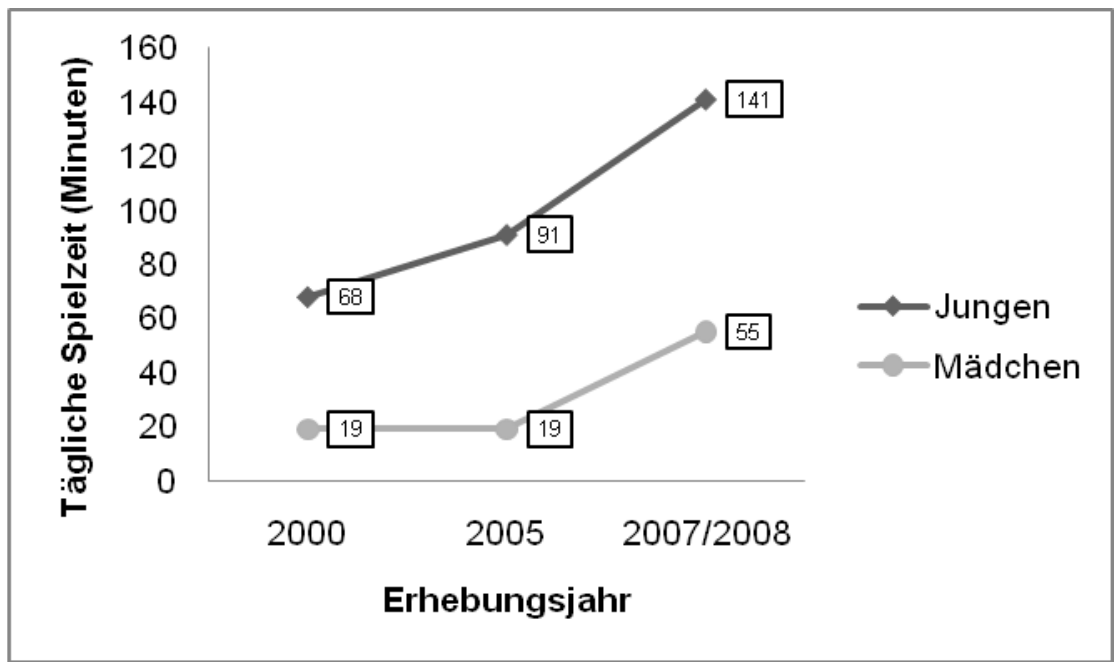

Abbildung 2. Tägliche durchschnittliche Computerspielnutzungszeit von Jugendlichen in den Jahren 2000, 2005 und 2007/2008 (eigene Berechnungen, vgl. Mößle, Kleimann, \& Rehbein, 2007; Rehbein, et al., 2009 a; Wilmers, et al., 2002) ${ }^{5}$

Wie die Ausstattungsquote variiert auch die Beschäftigungszeit mit Bildschirmmedien deutlich mit dem Lebensalter. Kleinkinder im Alter von zwei bis drei Jahren verbringen im Schnitt täglich 77 Minuten mit dem Fernsehen (Feierabend \& Mohr, 2004), Grundschulkinder im Alter von 10 Jahren ca. 90 Minuten (Mößle, et al., 2007) und Jugendliche im Alter von 15 Jahren rund 205 Minuten (Rehbein, et al., 2009 a). Sowohl im Kindes- als auch im Jugendalter ist Fernsehen unter allen medialen und nicht-medialen Freizeitaktivitäten die Beschäftigung mit der höchsten zeitlichen Relevanz (Mößle, et al., 2007; Rehbein, et al., 2009 a). Die hohe Nutzungsdauer des Mediums bleibt bis ins Erwachsenenalter bestehen. So weist der durchschnittliche Erwachsene (GFK Panel ab 14 Jahren) im Jahr 2007 eine tägliche Fernsehzeit von 223 Minuten auf (Feierabend \& Klingler, 2008). Das Computer-

5 Schülerbefragung $2000(\mathrm{~N}=10.460, M=15.2$ Jahre; drei westdeutsche Großstädte, eine ostdeutsche Großstadt und ein Landkreis), Frageformat: Geschätzte wöchentliche Nutzungszeit: "Wie viele Stunden verbringst du außerhalb der Schule pro Woche mit folgenden Aktivitäten: $\mathrm{Zu}$ Hause oder bei Freunden am Computer etwas machen oder Nintendo oder Ähnliches spielen?" (zu methodischen Details vgl. Wilmers, et al., 2002). Schülerbefragung 2005 ( $\mathrm{N}=14.301 ; M=15.1$ Jahre; zehn Städte und Regionen Westdeutschlands), Frageformat: Nutzungszeit von Computerspielen am Vortag der Befragung (vgl. Mößle, et al., 2007, S. 48 ff.). Schülerbefragung 2007/2008 ( $N=44.610 ; M=15.3$ Jahre; 61 bundesdeutsche Erhebungsgebiete), Frageformat: Geschätzte tägliche Nutzungszeit von Offline- und Onlinespielen (vgl. Rehbein, et al., 2009 a, S. 15). Zur Angleichung der Messzeitpunkte wurden Förderschüler aus allen Berechnungen einheitlich ausgeschlossen. 
spielen gewinnt ab dem Grundschulalter und damit später als das Fernsehen an Bedeutung. Zehnjährige spielen täglich etwa 30 Minuten an Computer oder Spielkonsole (Mößle, et al., 2007), Fünfzehnjährige rund 100 Minuten (Rehbein, et al., 2009 a). Wenngleich gerade im Jugendalter eine hohe Affinität zu Computerspielen besteht und Bildschirmmedien gerade in diesem Alter das Freizeitverhalten dominieren (vgl. Abbildung 3), nutzen auch Erwachsene Computerspiele. Dem Bundesverband Interaktive Unterhaltungssoftware zufolge sind 18 Prozent der Computerspielnutzer zwischen 20 und 29 Jahren alt und weitere 25 Prozent 30 Jahre und älter (Bundesverband Interaktive Unterhaltungssoftware e.V., 2009).

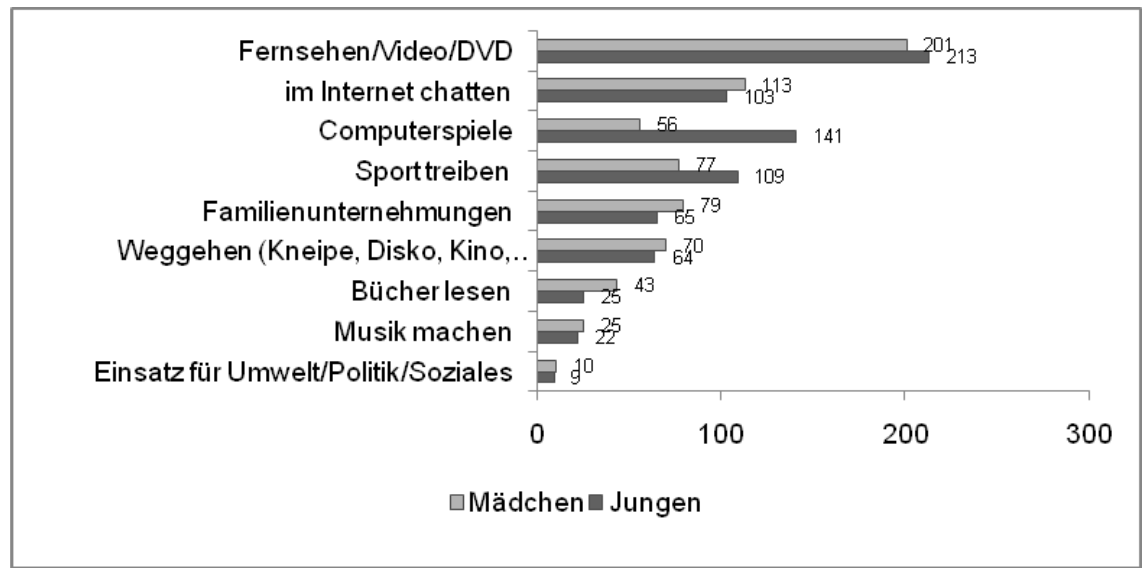

Abbildung 3. Das Freizeitbudget eines Neuntklässlers nach Geschlecht im Tagesdurchschnitt in Minuten $(n \approx 43.500$, gewichtete Daten, aus Rehbein et al., 2009)

Sowohl im Kindes- als auch im Jugendalter weist das männliche Geschlecht in Hinblick auf die Mediengeräteausstattung einen deutlichen Vorsprung auf (Feierabend \& Rathgeb, 2009 b; Mößle, et al., 2007; Rehbein, et al., 2009 a). Dies gilt insbesondere für Computer und Spielkonsole, in geringerem Maße auch für den Fernseher. Analog zeigt sich bei Jungen bereits im Grundschulalter eine höhere Gesamtnutzungszeit ${ }^{6}$ von Bildschirmgeräten mit 145 Minuten gegenüber Mädchen mit 96 Minuten. Dieser Vorsprung an täglicher Mediennutzungszeit bei den Jungen lässt sich sowohl für Sonntage als auch für Schultage nachweisen (Mößle, et al., 2007). Im Jugendalter wächst diese Differenz in der Gesamtmediennutzungszeit

6 Hierbei gilt zu bedenken, dass aus einer Summation von Medienzeiten Unschärfen resultieren, indem diese sich im Alltag von Kindern und Jugendlichen häufig überschneiden und so zum Beispiel das Fernsehen bei vielen Jugendlichen heute nur noch als „Nebenbei-Medium“ anderer medialer und nicht-medialer Tätigkeiten fungiert. 
auf 87 Minuten zugunsten der Jungen an (Rehbein, et al., 2009 a). Auch das Bildungsniveau im Elternhaus bestimmt im Kindesalter die Parameter Medienausstattung, Nutzungszeit und Nutzungsinhalte maßgeblich und ist zudem ein wichtiger Indikator des elterlichen Medienerziehungsverhaltens. So übersteigt die Computerausstattung von Viertklässlern mit niedrig gebildeten Eltern um 9 Prozentpunkte (42\% vs. $33 \%$ ), die Spielkonsolenausstattung um 32 Prozentpunkte (43\% vs. $11 \%$ ) und die Fernsehausstattung um 41 Prozentpunkte (57\% vs. 16\%) die Ausstattung von Viertklässlern mit hochgebildeten Eltern (Mößle, et al., 2007). Diese Unterschiede in Ausstattung, Nutzungszeit und der Nutzung entwicklungsbeeinträchtigender Angebote gleichen sich jedoch im Jugendalter gerade zwischen mittleren und höheren Bildungsniveaus deutlich an (Mößle, et al., 2007).

\subsubsection{Nutzung gewalthaltiger Medienangebote}

Fernseh- und Computerspielnutzung sind überwiegend unterhaltungsmotiviert. Ennemoser und Schneider (2007) klassifizieren auf Basis der Nutzungspräferenzen von 332 Grundschülern, die von der ersten bis zur vierten Klasse längsschnittlich begleitet wurden, einen Anteil von 75 Prozent der genutzten Programme als reine Unterhaltungs- und 25 Prozent als kognitiv förderliche Edutainmentformate. Der beliebteste Fernsehsender von Viertklässlern (Mößle, et al., 2007) sowie der Gruppe 3- bis 13-Jähriger (Feierabend \& Klingler, 2008) ist Super RTL, der hauptsächlich fiktionale Angebote - bestehend zu etwa 90 Prozent aus Trickfilmen - sowie weitere Unterhaltungsangebote bereithält, gleichzeitig aber den geringsten Anteil an Informationsprogrammen aufweist (Feierabend \& Klingler, 2008, S. 355). Im Gegensatz zu Super RTL mit dem höchsten Marktanteil unter 3 bis 13-Jährigen $(23,2 \%)$ fällt die Markrelevanz des stärker auf Edutainment abzielenden Senders KI.KA (13,8\%) geringer aus. Mit zunehmendem Lebensalter verlieren sowohl Super RTL als auch KI.KA zugunsten anderer privater Sender (insbesondere ProSieben, RTL) an Bedeutung (Feierabend \& Rathgeb, 2008). Die öffentlich-rechtlichen Sender verzeichnen hingegen mit dem Übergang vom Kindes- zum Jugendalter kaum Zugewinne (Feierabend \& Klingler, 2008). Auch bei der Computerspielnutzung gilt Unterhaltung als maßgebliches Nutzungsmotiv: So machten im Jahr 2005 unter den interaktiven Unterhaltungsangeboten Info- und Edutainmentangebote nur etwa 21 Prozent des Marktes aus (Bundesverband Interaktive Unterhaltungssoftware e.V., 2006).

Eine hohe Unterhaltungsorientierung geht jedoch nicht nur mit einer geringeren Nutzung von Lern- (Edutainment) und Informationsangeboten (Infotainment) ein- 
her, sondern auch mit einer häufigeren Nutzung gewaltbetonter ${ }^{7}$ Inhalte. Dies trifft insbesondere auf Jungen zu, die gewalthaltige Medien weit stärker präferieren als Mädchen (Gentile, Lynch, Linder, \& Walsh, 2004; Trudewind \& Steckel, 2003; van Schie \& Wiegman, 1997). Um eine Vorstellung von der Verbreitung von Gewalt in den Medien zu gewinnen bieten sich Inhaltsanalysen an, die sowohl zum Fernsehprogramm als auch zu Computerspielen durchgeführt wurden (vgl. Kunczik \& Zipfel, 2004, S. 18).

Eine der größten Studien zu Gewalt im Fernsehen ist die National Television Violence Study (NTVS), in welcher von 1994 bis 1997 die Angebote von 23 USamerikanischen Fernsehsendern einer qualitativen Inhaltsanalyse unterzogen wurden. Dabei sind zu jedem der insgesamt drei Messzeitpunkte etwa 2.700 Sendestunden erfasst und analysiert worden (Wilson, et al., 2002). Als ein zentrales Ergebnis der Studie zeigte sich, dass 57 Prozent (1994/1995) bis 61 Prozent (1996/1997) der Sendungen Gewalt ${ }^{8}$ enthalten (National Television Violence Study 1, 1997; National Television Violence Study 3, 1998). Auch Kontextfaktoren sowie der Realitätsgrad der Gewalt wurden in der Analyse berücksichtigt. 69 Prozent der Gewalthandlungen bestanden in wiederholter oder erweiterter Gewalt, indem die Angriffe mehr als eine Einzelsequenz umfassten (z. B. mindestens zweimal geschlagen oder geschossen). 39 Prozent der gewalthaltigen Interaktionen betrafen unmittelbare körperliche Gewalt, in weiteren 26 Prozent kamen Handfeuerwaffen (Pistolen) zum Einsatz und in 19 Prozent unkonventionelle Gegenstände wie Seile oder Stühle (National Television Violence Study 3, 1998). Es zeigte sich jedoch gleichfalls, dass Blut und Verletzungen nur selten visualisiert werden: Nur in 14 Prozent der Gewaltszenen fanden sich derartige Darstellungen in jeweils unterschiedlichem Schweregrad. Eine erneute Analyse der Daten der NTVS unter besonderer Berücksichtigung von Formaten für Kinder unter 12 Jahren zeigte, dass Kindersendungen einen höheren Anteil an Gewalthandlungen aufweisen (69\%) als Sendungen, die nicht an ein kindliches Publikum gerichtet sind (57\%). Insgesamt finden im Kinderfernsehen stündlich ca. 14 Gewalthandlungen statt. Der Anteil von tödlicher Gewalt und auch von wiederholter Gewalt gegen ein bestimmtes Opfer weist für die kindliche und nicht-kindliche Zielgruppe eine vergleichbare Auftretenshäufigkeit auf (52-62\%). Jedoch fällt im Kinderfernsehen der Anteil expliziter Gewalt im Sinne von deutlichen Blut- und Verletzungsdarstellungen

7 Zur Komplexität und Unschärfe des Mediengewaltbegriffs vgl. Imbusch (2002). Je nach Studie wird mit sehr unterschiedlichen Definitionen von Mediengewalt gearbeitet, die nachfolgend für jede Studie separat dargestellt werden. Die Ableitung der Mediengewaltdefinition dieser Untersuchung ist Kapitel 2.1.2.1 zu entnehmen.

8 Als Gewalt wurde eine glaubhafte Androhung oder tatsächliche Anwendung physischer Gewalt verstanden, wenn sie auf eine körperliche Beeinträchtigung eines anderen Lebewesens oder eine Gruppe von Lebewesen abzielt. Auch wenn nur die Konsequenzen einer vorausgegangenen Gewalthandlungen gezeigt wurden, wurde dies als Gewalt kodiert (National Television Violence Study 3, 1998, S. 30; Wilson, et al., 2002, S. 15). 
( $1 \%$ vs. $21 \%$ ) sowie die Häufigkeit mit der Waffen eingesetzt werden (9\% vs. $31 \%$ ) deutlich geringer aus. In Kindersendungen ist die Gewalt zudem weit häufiger in einen realitätsfernen Kontext wie etwa ein Fantasy- oder Trickfilmszenario eingebunden (Wilson, et al., 2002).

Für das deutsche Fernsehen gelangten Groebel und Gleich (1993) auf Basis einer Analyse von insgesamt sechs deutschen Fernsehsendern, aus deren Programm in einem Zeitraum von acht Wochen des Jahres 1991 eine Zufallsstichprobe von Sendungen gezogen wurde (insgesamt 750 Programmstunden), auf einen Anteil von etwa 50 Prozent von Sendungen, die aggressive Handlungen ${ }^{9}$ enthalten und auf eine Frequenz von fünf aggressiven Handlungen pro Stunde. Mehr als 50 Prozent der aggressiven Handlungen gingen mit physischen Schädigungen, 20 Prozent mit Mord/Tod einher. Zwischen 18:00 und 20:00 Uhr sowie nach 23:00 Uhr wurde eine besondere Häufung körperlicher Gewaltszenen registriert. Die Angebote der privaten Sender wiesen dabei einen deutlich höheren Anteil von aggressiven Ereignissen und Mordszenen auf als die der öffentlich-rechtlichen Sender. In einer aktuelleren inhaltsanalytischen Studie wurde in den Jahren 2002 und 2003 eine Stichprobe von 1.162 Programmstunden aus acht öffentlich-rechtlichen und privaten Vollprogrammen sowie den Spartenkanälen KI.KA und Super RTL gezogen und dabei der Gewaltantei ${ }^{10}$ von Sendungen im Tagesverlauf von 06:00 bis 23:00 Uhr analysiert (P. Grimm, et al., 2005). Etwa 30 der insgesamt 1.162 Programmstunden $(2,6 \%)$ entfallen auf konkrete Gewaltdarstellungen. Mehr als die Hälfte der analysierten Sendungen (58 \%) wiesen mindestens eine Gewaltdarstellung auf. Von den Gewaltsequenzen werden 19 Prozent visuell detailliert dargestellt. USamerikanische Sendungen, die einen Anteil von 28,9 Prozent am Gesamtangebot ausmachen, weisen dabei den größten Gewaltanteil auf. Der weit überwiegende Anteil von Gewalt (87\%) umfasst intentionale Gewalt, bei der demnach eine Absicht und Motivation des Angreifers unterstellt werden kann, körperliche Gewalt

9 In der Studie wird insgesamt ein weites Verständnis von "Aggression" angesetzt und verschiedene Aggressionsformen einbezogen. Verhaltensweisen werden als aggressiv bezeichnet, wenn "erkennbar eine Person oder eine Gruppe von Personen eine andere Person oder Gruppe sowie Tiere und Sachen bewußt und mit Absicht schädigt oder bei einer Handlung eine solche Schädigung in Kauf nimmt" (Groebel \& Gleich, 1993, S. 34). Aggressive Handlungen sind dabei nur solche, die von Personen oder menschenähnlichen Wesen ausgehen und dabei weder der Rettung einer Person dienen noch als übliche Handlungen innerhalb eines sportlichen Wettkampfes oder als humorvoller Einsatz von Scherzartikeln gelten können (Groebel \& Gleich, 1993, S. 41). Als aggressive Handlungen wurden auch schwere Bedrohungen gewertet (Groebel \& Gleich, 1993, S. 53). Zum Problem einer synonymen Verwendung des Aggressions- und Gewaltbegriffes in inhaltsanalytischen Medienstudien (vgl. P. Grimm, Kirste, \& Weiß, 2005, S. 43 ff.).

10 "Gewalt ist die physische, psychische oder materielle Schädigung von Objekt(en) durch Subjekt(e), Naturgewalten oder Unfälle. Gewalt kann intentional oder intentionsunabhängig sein. Intentionale Gewalt umfasst drei Einheiten: glaubwürdige Drohungen, die Gewalttat selbst sowie schädliche Folgen. Intentionsunabhängige Gewalt umfasst zwei Einheiten: den Vorfall und die schädlichen Folgen" (P. Grimm, et al., 2005, S. 64). 
(93\%), sowie Gewalt, die in realitätsnahe (36\%) oder reale Kontexte (34\%) eingebettet ist (im Vergleich fantastische Gewalt: $22 \%$ ). Die Gewaltakteure werden jedoch insgesamt häufiger als negativ (38\%) denn als positiv (17\%) charakterisiert. Die meisten Gewaltmittel sind unmittelbare körperliche Gewalt mit natürlichen Mitteln (34\%) und Handfeuerwaffen (ca. 19\%). Unter den geschilderten Gewaltfolgen dominieren körperliche Schäden (69\%). Von diesen wiederum entfällt ein Drittel auf extreme Schäden im Sinne lebensgefährlicher Verletzungen oder Tod und weitere 23 Prozent auf "schwere Gewaltfolgen, wie zum Beispiel tiefe Wunden oder ernsthafte Verbrennungen" (P. Grimm, et al., 2005, S. 119).

Auch für Computerspiele wurde auf einen hohen Anteil von Gewalthandlungen und -darstellungen hingewiesen. Dietz führte eine Inhaltsanalyse an 33 Videospielen der Firmen Nintendo und Sega durch, die im Jahr 1995 besonders populär und verbreitet waren. Nur 21 Prozent der analysierten Spiele enthielten keinerlei Formen von gewaltbezogenen oder aggressiven Handlungen. ${ }^{11}$ Nahezu die Hälfte der Spiele wies jedoch gegen andere Spielcharaktere gerichtete Gewalt und aggressive Handlungen auf. Häufig waren die Opfer menschenähnliche Wesen, die grafische Darstellung explizit und die Probleme im Spiel nur mit Gewalt zu lösen (Dietz, 1998). Smith, Lachlan und Tamborini (2003) zeigen in einer Untersuchung von 60 besonders verbreiteten Konsolenspielen aus dem Jahr 1999, dass hiervon 68 Prozent Gewalt ${ }^{12}$ enthielten. 27 Prozent wiesen besonders drastische Gewalt in Form grausamer Konsequenzen für das Gewaltopfer auf Spiele, die vom Entertainment Software Rating Board (ESRB) "ab 13 Jahren" und "ab 17 Jahren" freigegeben wurden, enthielten zu 90 Prozent Gewalt. Bei diesen Spielen wurden häufiger menschenähnliche Gegner präsentiert (78 \%) und im Schnitt 4,6 aggressive Handlungen pro Minute ausgeführt. Spiele für Kinder "ab 6 Jahren" beinhalteten zwar fiktionalere Spielgestalten und stellten weitaus seltener Blut und Splattereffekte dar, enthielten aber ebenfalls zu 57 Prozent Gewalt und wiesen 1,2 aggressive Handlungen pro Minute auf. Nur 10 Prozent der vom Spieler gesteuerten Protagonisten waren als "guter Spielcharakter" zu bewerten und wiesen prosoziale Eigenschaften auf. Thompson und Haninger (2001) untersuchten 55 Videospiele, die im April 2001 in den USA auf dem Markt verfügbar waren und vom Entertainment Software Rating Board (ESRB) als "Everyone (E)" eingestuft und damit als geeignet für Personen ab 6 Jahren befunden wurden. 64 Prozent dieser Spiele ent-

11 Die Spiele wurden danach unterschieden, ob sie: 1. Überhaupt keine Gewalt oder Aggression, 2. Sozial adäquate Formen von Aggressionen wie z. B. in Sportspielen, 3. Gewalt gegen Frauen oder 4. Gewalt gegen andere Personen enthielten (Dietz, 1998, S. 433).

12 Als Gewalt wurde - in Entsprechung zur NTVS-Studie (vgl. Wilson, et al., 2002, S. 15) eine glaubhafte Androhung physischer Gewalt oder eine tatsächliche Anwendung physischer Gewalt verstanden, wenn sie auf eine körperliche Beeinträchtigung eines anderen Lebewesens oder eine Gruppe von Lebewesen abzielt. Auch wenn nur die Konsequenzen einer vorausgegangenen Gewalthandlung gezeigt wurden, wurde dies als Gewalt kodiert (Smith, et al., 2003, S. 62 ff.). 
hielten zielgerichtete Gewalt ${ }^{13}$ gegen andere Wesen. Unter diesen gewalthaltigen Spielen nahmen Gewalthandlungen rund 30 Prozent der Spielzeit in Anspruch. In 60 Prozent der Spiele wurde Gewalt belohnt oder war erforderlich, um im Spiel voranzuschreiten. Nahezu die Hälfte der Spiele visualisierte Tötung als Folge von Gewalt. Obwohl die häufigste Gewalt unmittelbare körperliche Gewalthandlungen waren (55\%), enthielt eine Vielzahl der Spiele Waffen wie zum Beispiel Pistolen (24\%) oder Messer und Schwerter (11\%). Auch in einer weiteren Analyse der im Jahr 2001 besonders populären Spieltitel fanden sich in 48 Prozent der 70 analysierten Spiele ernsthafte Gewaltdarstellungen, weitere 39 Prozent enthielten comichafte Gewalt. ${ }^{14}$ In 41 Prozent der Spiele musste der Spieler Gewalt anwenden, um die Spielziele zu erreichen (Glaubke, et al., 2001).

Bei Computerspielen ist zudem aufgrund der technischen Weiterentwicklung der Spielehardware ein deutlicher Anstieg des Realitäts- sowie des Detaillierungsgrades der Gewaltdarstellungen zu konstatieren (vgl. Barlett \& Rodeheffer, 2009, S. 213; Gentile, et al., 2004, S. 8; Panee \& Ballard, 2002, S. 2459). So weisen Anderson und Bushman darauf hin, dass Videospiele zwar schon seit den 1970er Jahren verfügbar sind, jedoch erst seit den 1990er Jahren explizite "Tötungsspiele" wie Mortal Kombat, Street Fighter oder Wolfenstein 3D auf dem Markt seien, in denen das primäre Spielziel darin besteht zu verwunden oder zu töten und diese Handlungen audiovisuell zudem deutlich in Szene gesetzt werden (C. A. Anderson \& Bushman, 2001). Mit jeder neuen Spielgeneration ließe sich so ein Anstieg der Gewalt und ein Trend zu personalisierter Gewalt beobachten (Subrahmanyam, Kraut, Greenfield, \& Gross, 2001, S. 85). Hinsichtlich des deutschen Fernsehens kann die Frage, ob die Häufigkeit und Drastik von Gewaltdarstellungen insgesamt zugenommen hat, kaum beantwortet werden, da seit den frühen 1990er keine umfänglichen Inhaltsanalysen mehr durchgeführt wurden (P. Grimm, et al., 2005). Wenngleich die Studie von Petra Grimm und Kollegen zeigt (2005), dass gerade die in den letzten zehn Jahren entstandenen Reality-Formate einen hohen Gewaltanteil aufweisen, lässt sich hieraus noch kein Gesamtanstieg von Gewalt im deutschen Fernsehen ableiten.

Von mancher Seite wird eingewendet, dass eine objektive Bestimmung des Ausmaßes gewalthaltiger Unterhaltungsmedienformate auf Basis inhaltsanalyti-

13 Als Gewalt wurden Handlungen definiert, bei denen ein Aggressor anderen Charakteren willentlich körperliche Verletzungen zufügt oder sie tötet bzw. dies versucht. Kompetitive Handlungen im Rahmen sportlicher Wettkämpfe, Naturgewalten oder gefährliche Hindernisse ohne erkennbaren Aggressor wurden hingegen nicht als Gewalt kodiert (Thompson \& Haninger, 2001, S. 593).

14 Als comichaft wurde Gewalt dann eingestuft, wenn sie in einen humorvollen, spielerischen Kontext eingebunden war und mit geringen oder keinen Folgen für das Opfer verbunden war. Ernsthafte Gewalt wies demgegenüber eine höhere Dramatik und einen realistischeren Kontext auf und resultiert häufig in Schädigungen und Verletzungen des Opfers (Glaubke, Miller, Parker, \& Espejo, 2001, S. 8). 
scher Studien an einer einheitlichen und konsensfähigen Definition des Gewaltbegriffes (vgl. Hoppe-Graff \& Kim, 2002, S. 912) bzw. der einheitlichen Festlegung der Analyseeinheit "Gewalthandlung" (vgl. Kunczik \& Zipfel, 2004, S. 18) scheitern muss. Die dargestellten Analysen sprechen jedoch insofern eine eindeutige Sprache, als dass sie übereinstimmend einen hohen Anteil an aggressiven Handlungen und Gewalt im Fernsehen wie auch in Computerspielen resümieren. Wie gezeigt werden konnte werden gewalthaltige Medien dabei nicht nur erwachsenen Zielgruppen angeboten, sondern auch "kindgerecht" vermarktet, indem in Zeichentrickfilmen Gewalt zwar abstrakter und fiktionaler dargestellt wird, jedoch gleichsam eine hohe Gewaltdichte sowie problematische Legitimationen dieser Gewalt vorherrschend sind. Auch in Computerspielen, die laut Alterseinstufung ${ }^{15}$ als für Kinder geeignet gekennzeichnet werden, konnte ein hohes Maß an Gewalthandlungen festgestellt werden (Höynck, Mößle, Kleimann, \& Rehbein, 2007; zu von der USK eingestuften Spielen ab 12 Jahren vgl. Höynck, Mößle, Kleimann, Pfeiffer, \& Rehbein, 2007; zu von der ESRB eingestuften Spielen ab 6 Jahren [E; K-A) vgl. Smith, et al., 2003; Thompson \& Haninger, 2001). Hinzu kommt, dass Heranwachsende nicht selten Angebote nutzen, die erst für ein höheres Alter freigegeben sind. Unter computerspielenden Kindern im Alter von 6 bis 13 Jahren haben 30 Prozent (Jungen: 35 \%, Mädchen: $23 \%$ ) schon einmal Spiele gespielt, die nicht für ihr Alter geeignet sind (Feierabend \& Rathgeb, 2009 b), unter 12 bis 19-Jährigen 61 Prozent (Feierabend \& Rathgeb, 2008). Zur Frage, wie viele Kinder und Jugendliche solche Angebote regelmäßig nutzen, kann die Schülerbefragung 2005 des KFN herangezogen werden (Mößle, et al., 2007). Hier gaben 3 Prozent der Viertklässlerinnen und 21 Prozent der Viertklässler unter den drei aktuell präferierten Lieblingsspielen mindestens ein Spiel an, welches eine Freigabe ab 16 Jahren oder ab 18 Jahren aufweist. Neuntklässlerinnen nutzten zu 2 Prozent und Neuntklässler zu 27 Prozent mindestens ein Spiel mit einer Freigabe ab 18 Jahren. Für die Nutzung entwicklungsbeeinträchtigender Filme kann von ähnlichen Werten ausgegangen werden. Unter den Jugendlichen gaben rund 6 Prozent der Mädchen und 23 Prozent der Jungen an, regelmäßig gewalthaltige Filme ab 18 Jahren zu nutzen (Mößle, et al., 2007).

15 Diese werden weltweit in vielen Ländern durch unterschiedliche Kontrolleinrichtungen, häufig Selbstkontrolleinrichtungen der Industrie, festgelegt. In Deutschland sind dies für Computerspiele die Unterhaltungssoftware Selbstkontrolle (USK), für die Bewertung von Filmen die Freiwillige Selbstkontrolle der Filmwirtschaft (FSK). In den USA existiert für Computerspiele das Entertainment Software Rating Board (ESRB) und für Filme das Motion Picture Association of America (MPAA) sowie das Film Advisory Board (FAB).In Großbritannien ist sowohl für Filme als auch für Computerspiele das British Board of Film Classification (BBFC) zuständig. Computerspiele werden in vielen europäischen Ländern (nicht Deutschland) nach dem Pan-European Game Information System (PEGI) eingestuft, welches durch die Interactive Software Federation of Europe (ISFE) entwickelt und im Frühjahr 2003 eingeführt wurde. 
Folgende Erkenntnisse zur privaten Bildschirmmediennutzung können festgehalten werden:

- Insbesondere die Nutzung interaktiver Medien ist in den letzten 10 Jahren stark angestiegen.

- Da sich hinsichtlich der Nutzung des Fernsehens kaum Veränderungen ergeben haben, interaktive Unterhaltungsmedienzeit aber kontinuierlich zugenommen hat, ist insgesamt von einem maßgeblichen Anstieg der Bildschirmmedienzeit auszugehen. Medienaktivitäten binden den größten Zeitanteil im Freizeitmenu von 15-Jährigen.

- Bildschirmmediennutzung unter Heranwachsenden ist primär unterhaltungsmotiviert. Info- und Edutainmentangebote sind im Mediennutzungsmenu insgesamt unterrepräsentiert.

- Sowohl das Fernseh- als auch das Computerspielangebot weist ein hohes Maß an Gewalt und aggressiven Interaktionen auf. Beim Computerspielen ist insbesondere von einem qualitativen Anstieg der Gewalt auszugehen, indem der Gewaltrealismus und die Detailfülle der Darstellungen stark zugenommen haben. Die Präsentation von Gewalt in Computerspielen weist gegenüber dem Fernsehen eine weit höhere Ereignisfrequenz auf.

- Jungen weisen eine auffälligere Mediensozialisation auf, indem sie im Vergleich zu Mädchen früher mit eigenen Mediengeräten ausgestattet sind, höhere Nutzungszeiten aufweisen und häufiger entwicklungsbeeinträchtigende Angebote nutzen.

- Insbesondere hinsichtlich der Unterhaltungsmediennutzung im Kindesalter existieren maßgebliche bildungsmilieuspezifische Unterschiede, die jedoch mit höherem Lebensalter zunehmend nivelliert werden.

\subsection{Bildschirmmediennutzung und Schulleistung}

In diesem Kapitel wird dargestellt, inwieweit die Medienumwelt von Kindern und Jugendlichen in multifaktoriellen Modellen zur Erklärung von Schulleistungen berücksichtigt wird und welcher Stellenwert ihr dabei im Vergleich zu anderen Faktoren zugeschrieben wird (Kapitel 1.2.1). Daraufhin werden die empirische Forschung (Kapitel 1.2.2) sowie Erklärungshypothesen (Kapitel 1.2.3) zum Zusammenhang von Bildschirmmediennutzung und Schulleistung dargestellt. Der Forschungsstand sowie die Hypothesenlage bilden die Grundlage eines Arbeitsmodells, welches an der (bislang weitestgehend ungeklärten) Frage nach der Art des Zusammenhangs von Bildschirmmediennutzung und Schulleistung anknüpft (Kapitel 1.2.4). Hierauf basiert das Forschungsprojekt Mediennutzung und Schulleis- 